\title{
Oncofertility and Fertility Preservation Counseling in Iranian Women with Breast Cancer
}

\author{
(1) Ramesh OMRANIPOUR, 1 (D) Anahita BORJIAN, ${ }^{2}$ (D) Mohammad Amin BORJIAN, ${ }^{3}$ (I) Newsha NAZARIAN \\ 'Department of Surgery, Tehran University of Medical Science, Breast Disease Research Center, Tehran-Iran \\ ${ }^{2}$ Department of Surgery, Tehran University of Medical Science, Cancer Institute, Tehran-Iran \\ ${ }^{3}$ Department of Radiology, Iran University of Medical Science, Tehran-Iran \\ ${ }^{4}$ Department of Medicine Tehran Medical Branch of Islamic Azad University, Faculty of Medicine, Tehran-Iran
}

\begin{abstract}
OBJECTIVE
To introduce the importance of oncofertility and fertility preservation counseling in women with breast cancer and to discuss possible impacting factors regarding Iranian breast cancer female patients' participation in fertility preservation programs.
\end{abstract}

\section{METHODS}

In this study, 146 females with breast cancer were studied. The eligibility criteria were: female patients with breast cancer, to be in their reproductive age (between 14 to 45 years). Patients with at least one of the following criteria were excluded from this study: Having received chemo-radiotherapy before the current treatment, having received hormonal or pharmacological assisted reproductive treatments previously and unwillingness to participate in this study.

\section{RESULTS}

Out of 146 patients, oncofertility counseling was requested for 55 eligible patients. Oocyte-egg cryopreservation was successful in 16 patients $(29.10 \%)$. In four patients $(7.27 \%)$, the number of ovarian follicles was inadequate. For 10 patients $(18.18 \%)$, oncologists advised initiation of chemotherapy before completion of preservation programs, and 25 patients (45.45\%) decided not to continue their preservation programs. Out of 91 patients not receiving oncofertility counseling, 76 patients (83.50\%) declared that their family planning was completed, 11 patients $(12.10 \%)$ were in the early stages of breast cancer and did not require chemoradiotherapy, and four patients $(4.40 \%)$ were in metastatic stages. We also found that contrary to the number of living children of patients, neither age nor marital status has an impact on their decisions to participate in fertility preservation programs.

\section{CONCLUSION}

To improve the quality of lives of Iranian women suffering from breast cancer, it is of utmost importance to raise awareness of oncofertility and to investigate the reasons for the under-implementation of fertility preservation programs in cancer patients.

Keywords: Breast neoplasms; cryopreservation; fertility; neoplasms.

Copyright $\odot$ 2020, Turkish Society for Radiation Oncology 


\section{Introduction}

In the past decades, improvements in therapeutic protocols for cancer have increased the overall survival rate in the majority of patients.[1] However, such treatments may have negative impacts on survivors' lives in economical, psychological, social and biological ways. Fertility loss, which has a long-term impact on survivors' lives, is among these adverse effects.[2] Chemotherapy and radiotherapy may potentially cause loss of fertility in patients. [3] Some experts believe that the adverse effects of anti-cancer treatments on fertility are amongst the most pivotal role-players in patients' quality of life.[4] In recent years, a new term has been introduced in cancer treatment as "quality survival," and cancer treatment protocols have focused more than ever on how the patients' lives will be after their treatment, rather than to just how long they live.[5,6] Oncofertility is a rapidly advancing field, discussing fertility preservation methods and other related topics in cancer patients.[3] This interdisciplinary science, intersecting reproductive medicine and oncology, has gained attention in recent years. [1,7] The primary objective of oncofertility is to provide cancer patients with provisional fertility counseling and fertility preservation options to improve their quality of life after receiving required cancer treatments.[8] In the U.S., breast cancer is the most common malignancy in females and approximately $12 \%$ of breast cancers are diagnosed in women who are younger than 44 years. [9] Breast cancer is one of the most prevalent malignancies among Iranian women. Furthermore, studies show that breast cancer in Iranian women is diagnosed about one decade earlier compared to their counterparts in western countries.[10] Established fertility preservation options for women with breast cancer include oocyte or embryo cryopreservation, ovarian tissue cryopreservation and inhibition of ovarian function using $\mathrm{GnRH}$ agonists. [11] Based on recent studies, reproductive age for cancer patients in whom family planning might be incomplete, range from 14 to 45 years. [4]

In Iran, for females with breast cancer whose family planning is incomplete, oncofertility counseling is requested between the diagnosis of breast cancer and commencing neoadjuvant or adjuvant chemotherapy.

The objective of the present study is to introduce the importance of oncofertility and fertility preservation counseling in women with breast cancer and to discuss possible impacting factors regarding Iranian breast cancer patients' participation and the result of fertility preservation programs in them.

\section{Materials and Methods}

From March 2017 to July 2019, eligible breast cancer female patients referred to our clinic were presented with oncofertility and fertility preservation options and then were requested to participate in our study. The eligibility criteria were: female patients with breast cancer, to be in their reproductive age (between 14 to 45 years of age). Patients with at least one of the following criteria were excluded from this study: Having received chemo-radiotherapy before the current treatment, having received previous pharmacological hormonal or medicinal assisted reproductive treatment previously and unwillingness to participate in this study. Overall, 146 patients participated in our study. Before initiation of data gathering for each patient, written informed consent was obtained from them, indicating that their treatment would continue regardless of their decision to collaborate and remain in this study. Absolute anonymity was respected during all stages. For statistical analysis, we used Microsoft ${ }^{\oplus} \mathrm{Ex}-$ $\operatorname{cel}^{\odot}$ (Version 1907) $\mathrm{IBM}^{\odot}$ SPSS $^{\odot}$ (Version 22.0.0.0) and tests were performed with the $95 \%$ confidence interval.

\section{Results}

Study subjects ranged from 20 to 42 years of age (mean age $=36.5 \pm 3.7$ years). Out of 146 patients, 105 patients were married $(71.9 \%)$, and 41 were single (28.1\%) (Fig. 1).

Cancer stages in the studied patients included: Stage 0 (in-situ) in 7 patients (4.79\%). Stage I in 37 patients $(25.34 \%)$. Stage II in 89 patients $(60.95 \%)$. Stage III in 9 patients $(6.16 \%)$. Stage IV in 4 patients $(2.73 \%)$.

Cancer pathologies in the studied patients included: Invasive ductal carcinoma (IDC) in $130 \mathrm{pa}$ tients (89.04\%). Invasive lobular carcinoma (ILC) in 7

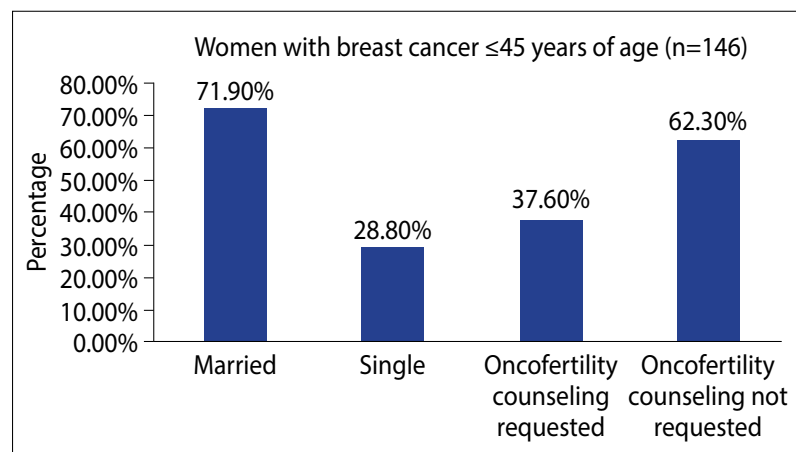

Fig. 1. Marital status and oncofertility counseling request a percentage of study subjects. 
patients (4.79\%). Ductal carcinoma in-situ (DCIS) in 7 patients (4.79\%). Invasive mucinous carcinoma in 2 patients (1.36\%).

Oncofertility and fertility preservation options were presented to all patients before the beginning of their cancer treatment. However, in 91 patients $(62.30 \%$ of all studied patients), oncofertility counseling was not requested for the following reasons; 76 patients $(83.5 \%)$ declared that their family planning is already completed, and they were unwilling to have more children (51 patients in stage II (67.11\%), 21 patients in stage I (27.63\%), 2 patients in stage III (2.63\%) and 2 patients in stage 0 (2.63\%). In 11 patients (12.1\%), due to breast cancer being in the early stages, chemotherapy was not required (5 patients in stage 0 (45.5\%), 4 patients in stage II in whom chemotherapy plan was canceled after oncotype DX $(36.36 \%)$ and 2 patients in stage I (18.18\%)). Therefore, the oncofertility counseling option was only introduced to them if they require it in the future. Four patients (4.4\%) had metastatic breast cancer (stage IV). Thus, the priority of cancer treatment to oncofertility counseling was explained to them, and subsequently, they decided to initiate their neoadjuvant therapy. Consequently, for 55 patients (37.70\% of all studied patients), oncofertility counseling was requested before the initiation of their therapy (Figs. 1 and 2).

Out of 55 consulted patients, in 16 patients (29.1\%), oocyte or egg cryopreservation was successfully performed (10 patients in stage II (62.5\%), 4 patients in

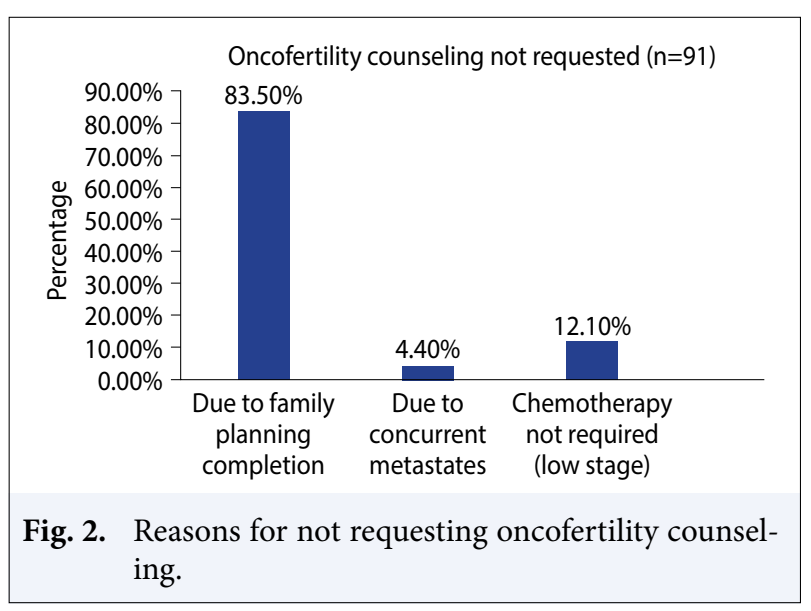

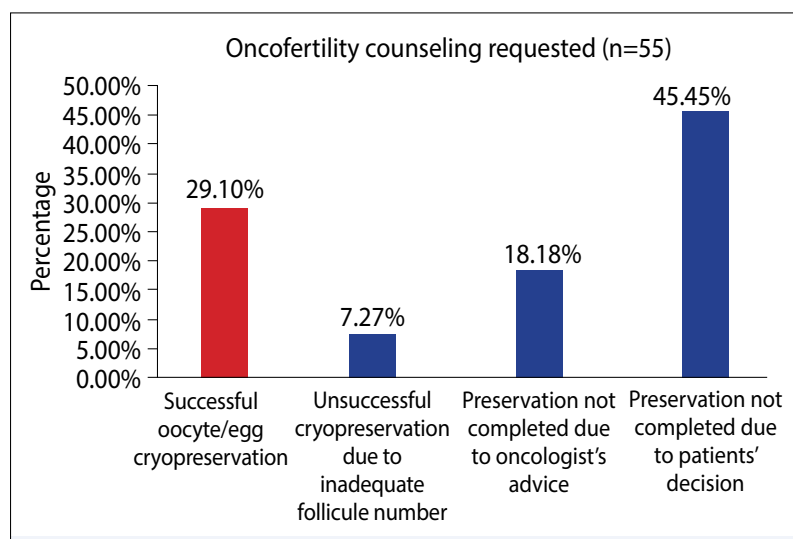

Fig. 3. Details of fertility preservation program completion.

stage I (25\%) and 2 patients in stage III (12.5\%)). In these patients, the average time, from the first day of oncofertility consultation to the completion of oocyteegg cryopreservation was 18.3 days (ranging from 12 to 30 days). In four patients (7.27\%), due to an inadequate number of ovarian follicles, cryopreservation of oocyte or egg was unsuccessful (all in stage II). Because the time-consuming process of cryopreservation (which was approximately 21 days, according to previous reports of the referral fertility preservation center) could delay systemic therapy, in ten patients (18.18\%) fertility preservation program was halted by the order of oncologist (8 patients in stage II (80\%) and 2 patients in stage I (20\%)). Lastly, 25 patients (45.45\%) did not complete the fertility preservation program (12 patients in stage II (48\%), 8 patients in stage I (32\%) and 5 patients in stage III (20\%)), declaring that they decided to allocate their financial resources to their breast cancer treatment, as

\begin{tabular}{|c|c|c|c|}
\hline & \multicolumn{2}{|c|}{ Decision } & \multirow[b]{2}{*}{ Total } \\
\hline & Decision: Yes & Decision: No & \\
\hline \multicolumn{4}{|c|}{ Marital status } \\
\hline Single & 20 & 12 & 32 \\
\hline Married & 10 & 13 & 23 \\
\hline Total & 30 & 25 & 55 \\
\hline
\end{tabular}

Table 1 Age-decision cross tabulation analysis

Decision n

Age

Decision: Yes

Decision: No

30

25

\section{Mean} SD

35.0333

36.0000
3.68111

3.35410
Std. Error Mean

0.67208

0.67082 
the fertility preservation costs were not covered by the insurance companies (Fig. 3).

We also tested two hypotheses using Chi-Square tests. The first hypothesis was to evaluate whether the age of the illegible patients has an impact on their decision to receive oncofertility consult before starting their treatment. We found that mean age in patients who decided not to receive consult was 36 years ( \pm 3.3 years), while mean age in patients who showed a desire to receive consult was 35 years ( \pm 3.6 years), which were not significantly different ( $\mathrm{p}$-value 0.31 ) (Table 1).

Another hypothesis was that marital status could affect patients' preference in receiving oncofertility consult. The analysis demonstrated that a larger proportion of unmarried patients preferred receiving counseling. However, between these two groups, the difference was not statistically significant ( $\mathrm{p}$ value $=0.182)($ Table 2$)$.

\section{Discussion}

Despite the importance of fertility preservation in cancer patients, oncofertility programs are mostly under-implemented, as there are multiple barriers blocking the wide usage of them. Some of these obstacles include lack of effective referral pathways and inefficient collaboration between clinicians, unaffordable costs, lack of public health awareness, shortage of oncofertility specialist and lack of universal preferred information delivering protocols.[3] For instance, since 2012, specific oncofertility guidelines have been implemented in Italy. These guidelines imply that oocyte cryopreservation, ovarian tissue cryopreservation and inhibition of gonads suing gonadotropin-releasing hormone agonists are offerable to patients. However, data regarding the actual needs for such services and the reason behind patients' refusal are still lacking.[12]

There are some studies about oncofertility in developing countries which show the shortage of knowledge of this field, alongside other obstacles in the way of implementing its guidelines (including high expenses of oncofertility services, oncologists' lack of awareness, cultural restraints and negative attitudes towards fertility preservation methods and clinicians and patients' tendency to allocate all their financial resources to their cancer treatment instead of fertility preservation programs). These findings raise awareness when the data show that approximately $50 \%$ of cancer patients in developing countries are below 65 years old and many are in their child-bearing age.[1] However, oncofertil- ity practice is growing in these countries despite all the mentioned problems.[13]

Cancer is the second most prevalent non-communicable disease in Iran [14], with an annual incidence of 33.1 per 100.000.[15] A study by Tazhibi et al. has shown that the incidence rate of breast cancer has increased from 2001 to 2010 in patients younger than 44 years of age.[16] The current treatment options of breast cancer may potentially be harmful to the fertility of the patients. [9] Despite these noticeable numbers and expanding deployment of oncofertility and fertility preservation protocols in Iranian institutes in recent years, limited research has been conducted on the importance of oncofertility and preservation options of newly diagnosed breast cancer female patients.

It has been shown that $75 \%$ of women in reproductive age who are diagnosed with cancer are interested in childbearing.[17] However, we found that in the majority of our patients, the fertility preservation programs were either not initiated or they were halted before completion. The findings are discussed in the following paragraphs.

In our study, the majority of studied patients did not receive oncofertility counseling from the beginning. (62.3\% of all patients) This was mainly because they declared that their family planning had already been completed, and they were not planning to have any more children in the time of the study $(83.5 \%$ of patients not receiving counseling). We suspect that stressful situations related to cancer diagnosis and its upcoming treatment could be a contributory factor in patients' decisions about participating in fertility preservation programs, which is a matter that may have negative impacts on their quality of life after completing their cancer treatment.

Our study also showed that 25 out of 55 consulted patients $(45.45 \%)$ decided to prioritize their cancer treatment and did not continue their fertility preservation program. We suspect that similar to studies from other developing countries $[1,13]$, the main reason behind this finding could be their decision to allocate all their financial resources to cancer treatment rather than to spend a part of it on fertility preservation programs. It is also important to mention that fertility preservation expenses are not covered by the insurance companies in Iran.

Another contributory factor could be the lack of adequate awareness in patients or social negative attitude towards the cryopreservation of oocyte or egg.

All the mentioned possible factors require detailed confirmatory studies in the future. 
Regarding the success of fertility preservation programs, we found the following results. Among the 55 patients referred to fertility preservation institutes, in four patients, (7.27\%) cryopreservation was not successful because of inadequate ovarian follicle numbers. Further studies and clinical trials are required to evaluate if adequate follicle numbers could be achieved in women with cancer using pharmacologic or hormonal therapies before referring them to fertility institutes, and subsequently, increase the rate of success of fertility preservation programs.

Similarly, in the aforementioned group, in 10 patients (18.18\%) fertility preservation program was halted due to oncologists' orders (the reason for which was the time needed to complete the fertility preservation programs would delay anticancer treatment). Regarding this matter, recent studies have shown that delaying anti-cancer therapy is not necessary when using ovarian stimulation protocols.[18-20] Also, ovarian stimulation protocols have not shown to have a negative effect on patients' prognosis.[20,21] Thus, it is important to discuss these findings with oncologists and to plan more comprehensive studies to reach a unified guideline.

We also found that neither age nor the marital status of breast cancer patients affects their decision to receive oncofertility counseling before starting their treatment. However, these findings could be biased due to rather a small number of studied patients and require further evaluation in future studies.

In addition, studies have shown that the number of live children could impact patients' desires and decisions, and patients who have at least one live child are less likely to be determined about participating in fertility preservation programs. [22,23] In our study, we found that all married patients who claimed their family planning had been completed, at least had one living child. This finding is compatible with the mentioned studies.

\section{Conclusion}

In conclusion, oncofertility remains a novel topic in Iranian medical literature. With Iran being among the countries with a high prevalence of cancers, it is of utmost importance to raise awareness of clinicians and patients of this topic, as well as introduce it to governmental and insurance companies to provide infrastructures of funding fertility preservation programs. We hope this study becomes the first step in solving the aforementioned barriers and improving the quality of life of Iranian women suffering from breast cancer and their families.
Peer-review: Externally peer-reviewed.

Conflict of Interest: Authors declare no conflicts of interest in the preparation for the present study.

Ethics Committee Approval: This study was approved by Breast Disease Research Center Medical Ethics Committee.

Financial Support: All costs for this study were covered by the authors, and no external funding resources were used.

Authorship contributions: Concept - R.O.; Design - A.B.; Supervision - R.O.; Funding - R.O.; Materials - R.O.; Data collection and/or processing - A.B.; Data analysis and/or interpretation - A.B., M.A.B.; Literature search - N.N.; Writing - M.A.B.; Critical review - R.O.

\section{References}

1. Salama M, Ataman L, Taha T, Azmy O, Braham M, Douik F, et al. Building Oncofertility Core Competency in Developing Countries: Experience From Egypt, Tunisia, Brazil, Peru, and Panama. J Glob Oncol 2018;4:1-11.

2. Vesali S, Navid B, Mohammadi M, Karimi E, OmaniSamani R. Little information about fertility preservation is provided for cancer patients: A survey of oncologists' knowledge, attitude and current practice. Eur J Cancer Care (Engl) 2019;28(1):e12947.

3. Anazodo A, Laws P, Logan S, Saunders C, Travaglia J, Gerstl B, et al. How can we improve oncofertility care for patients? A systematic scoping review of current international practice and models of care. Hum Reprod Update 2019;25(2):159-79.

4. Logan S, Perz J, Ussher JM, Peate M, Anazodo A1. A systematic review of patient oncofertility support needs in reproductive cancer patients aged 14 to 45 years of age. Psychooncology 2018;27(2):401-9.

5. Hovatta O. Cryopreservation of testicular tissue in young cancer patients. Hum Reprod Update 2001;7(4):378-83.

6. Reebals JF, Brown R, Buckner EB. Nurse practice issues regarding sperm banking in adolescent male cancer patients. J Pediatr Oncol Nurs 2006;23(4):182-8.

7. Quinn GP, Vadaparampil ST, Gwede CK, Miree C, King LM, Clayton HB, et al. Discussion of fertility preservation with newly diagnosed patients: oncologists' views. J Cancer Surviv 2007;1(2):146-55.

8. Rashedi AS, de Roo SF, Ataman LM, Edmonds ME, Silva AA, Scarella A, et al. Survey of Fertility Preservation Options Available to Patients With Cancer Around the Globe. J Glob Oncol 2018;4:1-16.

9. Petersen LF, Moravek M, Woodruff TK, Jeruss JS. Oncofertility Options for Young Women With Breast Cancer. The Breast. Elsevier Inc; 2018. p. 773-7. 
10. Mousavi SM, Montazeri A, Mohagheghi MA, Jarrahi AM, Harirchi I, Najafi M, et al. Breast cancer in Iran: an epidemiological review. Breast J 2007;13(4):38391.

11. Kim H, Kim SK, Lee JR, Hwang KJ, Suh CS Kim SH. Fertility preservation for patients with breast cancer: The Korean Society for Fertility Preservation clinical guidelines. Clin Exp Reprod Med 2017;44(4):181-6.

12.Lambertini M, Fontana V, Massarotti C, Poggio F, Dellepiane $\mathrm{C}$, Iacono $\mathrm{G}$, et al. Prospective study to optimize care and improve knowledge on ovarian function and/or fertility preservation in young breast cancer patients: Results of the pilot phase of the PREgnancy and FERtility (PREFER) study. Breast 2018;41:51-6.

13. Salama M, Ataman-Millhouse L, Sobral F, Terrado G, Scarella A, Bourlon MT, et al. Barriers and Opportunities of Oncofertility Practice in Nine Developing Countries and the Emerging Oncofertility Professional Engagement Network. J Glob Oncol 2018;4:1-7.

14. Dolatkhah R, Somi MH, Kermani IA, Ghojazadeh M, Jafarabadi MA, Farassati F, et al. Increased colorectal cancer incidence in Iran: a systematic review and meta-analysis. BMC Public Health 2015;15:997.

15. Nafissi N, Khayamzadeh M, Zeinali Z, Pazooki D, Hosseini M, Akbari ME. Epidemiology and Histopathology of Breast Cancer in Iran versus Other Middle Eastern Countries \%J Middle East Journal of Cancer 2018;9(3):243-51.

16. Tazhibi M, Dehkordi ZF, Babazadeh S. Trends in breast cancer incidence rates by age and tumor characteristics of women in the city of Isfahan for the period
2001-2010: An application of joinpoint analysis. J Res Med Sci 2014;19(4):319-25.

17. Geue K, Richter D, Schmidt R, Sender A, Siedentopf F, Brähler E, et al. The desire for children and fertility issues among young German cancer survivors. J Adolesc Health 2014;54(5):527-35.

18. Lee S, Ozkavukcu S, Heytens E, Moy F, Oktay K. Value of early referral to fertility preservation in young women with breast cancer. J Clin Oncol 2010;28(31):4683-6.

19. Letourneau JM, Sinha N, Wald K, Harris E, Quinn M, Imbar $\mathrm{T}$, et al. Random start ovarian stimulation for fertility preservation appears unlikely to delay initiation of neoadjuvant chemotherapy for breast cancer. Hum Reprod 2017;32(10):2123-9.

20. Chien AJ, Chambers J, Mcauley F, Kaplan T, Letourneau J, Hwang J, et al. Fertility preservation with ovarian stimulation and time to treatment in women with stage II-III breast cancer receiving neoadjuvant therapy. Breast Cancer Res Treat 2017;165(1):151-9.

21. Azim AA, Costantini-Ferrando M, Oktay K. Safety of fertility preservation by ovarian stimulation with letrozole and gonadotropins in patients with breast cancer: a prospective controlled study. J Clin Oncol 2008;26(16):2630-5.

22. Yee S, Fuller-Thomson E, Lau A, Greenblatt EM. Fertility preservation practices among Ontario oncologists. J Cancer Educ 2012;27(2):362-8.

23. Goodman LR, Balthazar U, Kim J, Mersereau JE. Trends of socioeconomic disparities in referral patterns for fertility preservation consultation. Hum Reprod 2012;27(7):2076-81. 